\title{
Mixed Medullary-Follicular Carcinoma and Papillary Carcinoma of the Same Thyroid
}

\author{
Chung-Jung $\mathrm{WU}^{*}, * * *$, Hsiang-Ling CheN ${ }^{* *}$, Yuh-Min Song*, Wayne Huey-Herng SHeU* and Shin-Ho CheN $* *$
}

We report a rare case of mixed medullary-follicular carcinoma and papillary carcinoma of the same thyroid. A 27-year-old Chinese female complained of a single thyroid nodule for 2-3 months. Needle aspiration revealed suspicious papillary carcinoma and thyroidectomy performed later showed mixed medullary-follicular carcinoma and papillary carcinoma of the same thyroid which was extremely rare. Whether neoplastic transformation is due to tumorigenic stimulus or just due to the collision phenomenon is still controversial for its etiology.

(Internal Medicine 37: 955-957, 1998)

Key words: thyroid, follicular carcinoma, papillary carcinoma, medullary carcinoma, calcitonin, psamomma body, neck nodule

\section{Introduction}

Primary carcinomas of the thyroid are often reported as papillary carcinoma, follicular carcinoma and medullary carcinoma individually or in a collision pattern. Frequently, medullary carcinoma is associated with follicular carcinoma and thus termed as mixed medullary-follicular carcinoma of the thyroid. Although the coexistence of mixed medullary-follicular carcinoma and papillary carcinoma has been reported, all in the same lobe of the thyroid is very rare.

For editorial comment, see p 909.

\section{Case Report}

A 27-year-old Chinese female complained of a right neck nodule and mild dysphagia for 2-3 months. There was no family history of hypertension or thyroid disorders, including medullary carcinoma. Physical examination revealed only a single nodule, about $1 \mathrm{~cm}$ in diameter, over the right lobe of the thyroid without evidence of mucosal neuroma. A non-functioning nodule was demonstrated on Tc-99m thyroid scan. Fine needle aspiration revealed suspicious papillary carcinoma of the thyroid (Fig. 1).

Thyroidectomy was performed with the finding of a firm, solid tumor of $1.5 \times 1 \times 1 \mathrm{~cm}$ in size over the right lobe of the thyroid. The cut surface was red-brownish with firm consistency. Histologically, it was composed of a larger nodule of mixed medullary-follicular carcinoma. The components of medullary carcinoma were polygonal and spindle clear cells, which were positively stained for calcitonin, weakly positive for chromogranin A, and focally positive for thyroglobulin after PAP staining. No amyloid deposition was found (Fig. 2). The cells of follicular carcinoma had round to oval nuclei with fine chromatin and were arranged in a solid and microfollicular

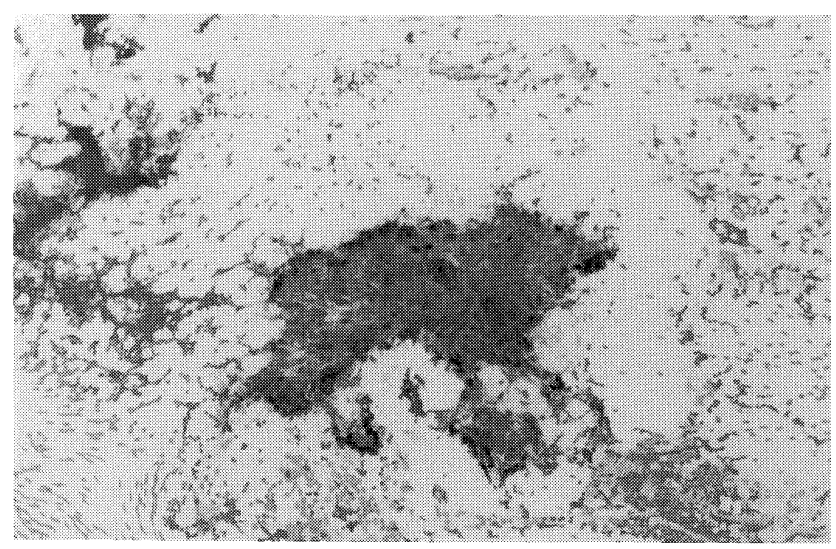

Figure 1. Suspicious papillary carcinoma of the thyroid on fine-needle aspiration. It may be truly a medullary carcinoma (HE stain, $\times 100$ ).

From *the Division of Endocrinology and Metabolism in Department of Medicine, **the Department of Pathology, Taichung Veterans General Hospital, Taichung, ***Tzu Hui Senior Vocational School of Nursing and Midwifery, Pingtung, Taiwan

Received for publication March 17, 1998; Accepted for publication August 11, 1998

Reprint requests should be addressed to Dr. Chung-Jung Wu, the Division of Endocrinology and Metabolism, Taichung Veterans General Hospital, No. 160, Section 3, Chung-Kang Road, Taichung 407, Taiwan, ROC 

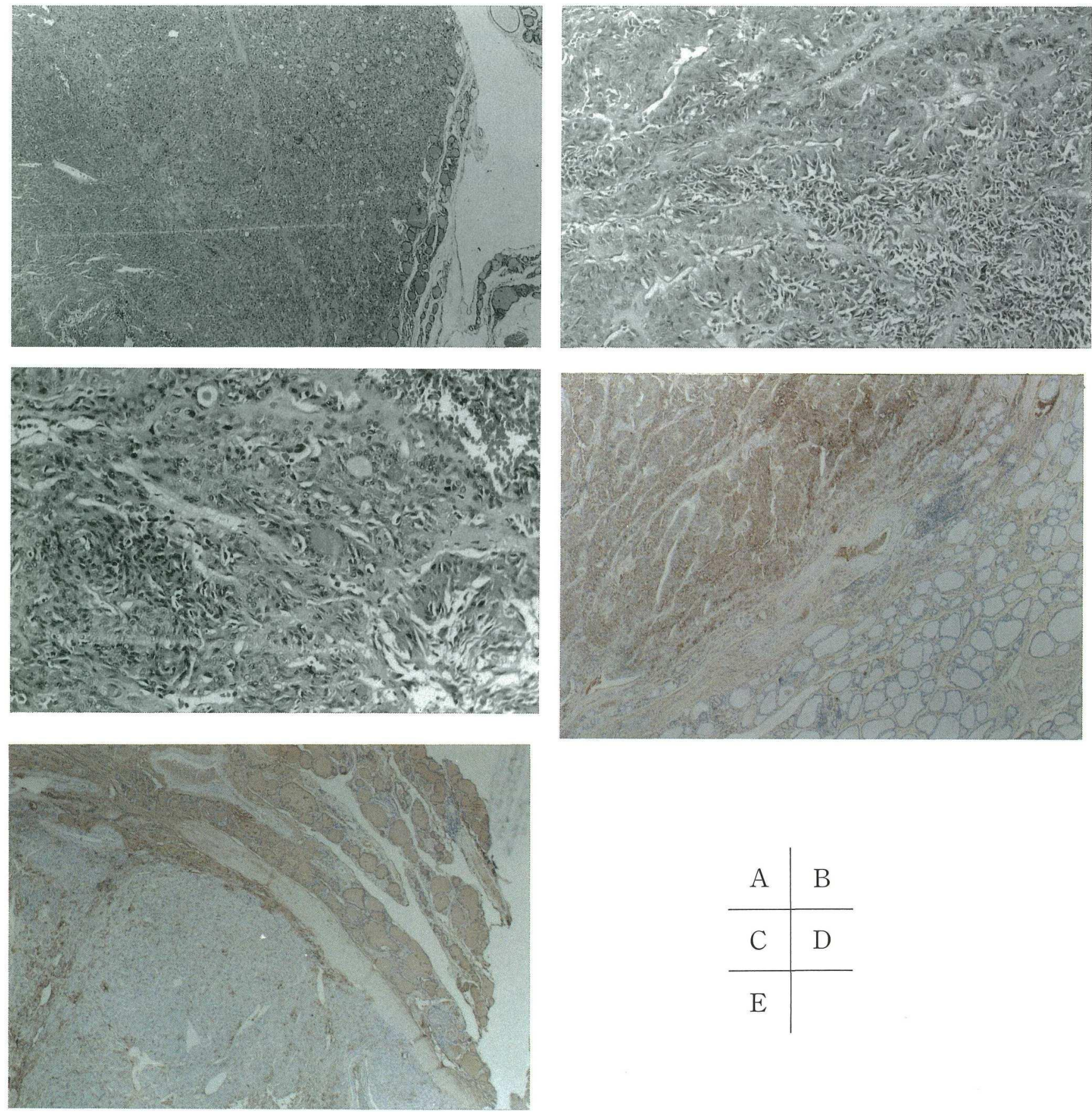

Figure 2. Mixed medullary-follicular carcinoma (A: HE stain, $\times 40$ ) was composed of polygonal or spindle shape clear cells with nearly no amyloid deposition (B: HE stain, $\times 200$ as from left lower area of $A)$ and microfollicle formation $(\mathrm{C}: \mathrm{HE}$ stain, $\times 200$ as from central area of A). Calcitonin stain was strongly positive in medullary carcinoma (D: HE stain, $\times 40)$. Thyroglobulin stain was negative in medullary carcinoma but positive in the follicular area $(\mathrm{E}: \mathrm{HE}$ stain, $\times 40)$.

pattern (Fig. 3). A small nodule of about $0.1 \mathrm{~cm}$ in diameter as shown from frozen section revealed papillary carcinoma with overlapping atypical nuclei and psamomma bodies in the same lobe (Fig. 4). Thus mixed medullary-follicular carcinoma and papillary carcinoma of the same thyroid was diagnosed.

She received total thyroidectomy followed by radioiodine irradiation later. Postoperative follow up of tumor markers such as carcinoembryonic antigen (CEA) (before surgery and follow up: $23.73 \mathrm{ng} / \mathrm{ml}$ and $1.7 \mathrm{ng} / \mathrm{ml}$ ) and calcitonin (not checked before surgery; follow-up was $46.95 \mathrm{ng} / \mathrm{dl}$ ) were all in the normal range. There has been no evidence of recurrent disease for 9 months to date. 


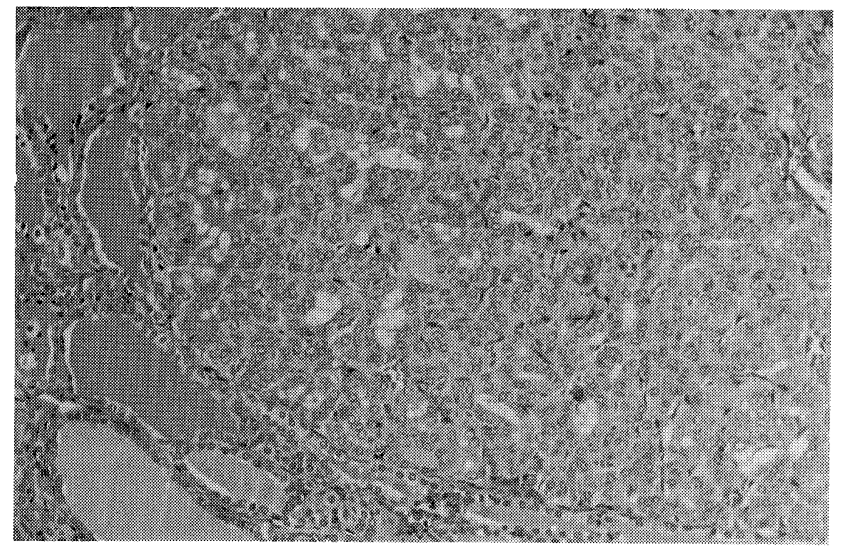

Figure 3. Follicular carcinoma was around by the normal follicular area and high power view showed round to oval cells with fine chromatin and aggregation of micro-follicular pattern (HE stain, $\times 200$ ).

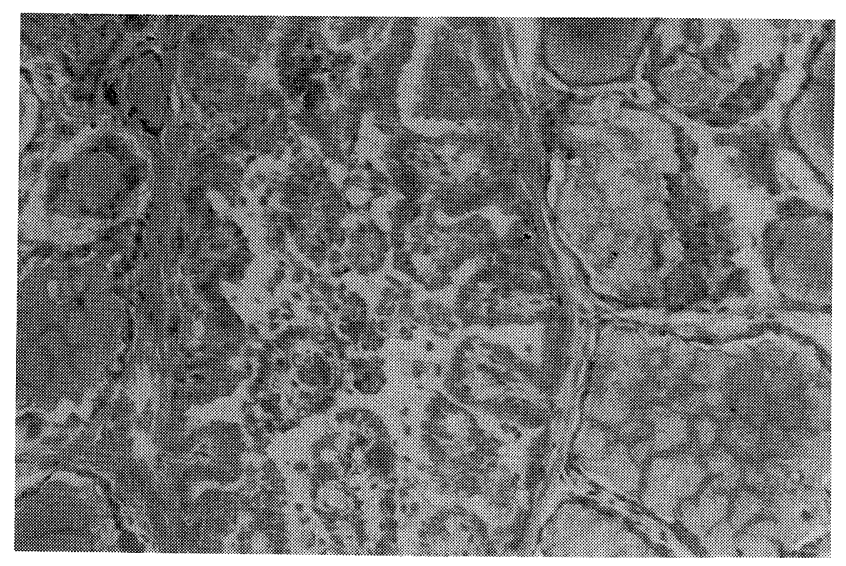

Figure 4. Papillary carcinoma with psamomma body in papillary projection and few eosinophilic inclusion bodies are found (HE stain, $\times 150)$.

\section{Discussion}

It has been known that medullary carcinoma of the thyroid is frequently associated with multiple endocrine neoplasia (MEN) type IIa, as pheochromocytoma and parathyroid gland hyperplasia; or type IIb, as pheochromocytoma and mucosal neuroma or even familial medullary thyroid cancer (FMTC). All hereditary medullary carcinomas of the thyroid are bilaterally involved. But sporadic cases also have been reported. In this case, there was no aforementioned associations of multiple endocrine neoplasia, familial history or bilaterally involved thyroid gland. Thyroid tissue is composed of thyroidal cell (follicular cell) and parafollicular cell (C-cell) which originate from different embroynic sources, endoderm and ectoderm, respectively. Mixed medullary-follicular carcinoma of the thyroid has been categorized as a separate group and more than a dozen cases have been reported $(1,2)$. Although mixed medullary-follicular carcinoma and papillary carcinoma of the thyroid have been reported, they are not typical. In the cases reported by Michal et al, only rare follicles, that were cytologically identical cells of papillary carcinoma, were found in mixed medullary-follicular carcinoma without existence of psamomma body (3). Parker et al reported a case of mixed medullary, papillary, follicular and undifferentiated pattern of thyroid carcinoma in metastatic lymph node lesion without showing mixed medullary-follicular carcinoma (4). But the reports of mixed medullary-follicular carcinoma and papillary carcinoma of the same thyroid in single nodule (less than $2 \mathrm{~cm}$ in size) are rare. The combination of thyroid carcinomas in this case is extremely rare. It has been proposed that the coincidence of these carcinomas arises from separate embryologic origins (5). In this case, medullary carcinoma is free of amyloid deposition with abnormal product accumulation of chromogranin A. Thus the transformation of thyroid cells (thyroidal follicular cell and parafollicular cell) to neoplastic change due to uncertain episodes such as tumorigenic stimulus may be an alternative explanation. But mixed medullary-follicular carcinoma with the coexistence of thyroid papillary carcinoma can not be ruled out as there is a high reported incidence of micropapillary carcinoma of the thyroid in autopsy.

\section{References}

1) Kos M, Separovic V, Sarcevic B. Medullary carcinoma of the thyroid: histomorphological, histochemical, and immunohistochemical analysis of twenty cases. Acta Med Croatica 49: 195-199, 1995.

2) Papotti M, Negro F, Carney JA, Bussolati G, Lloyd RV. Mixed medullary-follicular carcinoma of the thyroid. A morphological, immunohistochemical and in situ hybridization analysis of 11 cases. Virchows Arch 430: 397-405, 1997.

3) Michal M, Curik R, Macak J, Ludvikova M, Dedic K. Mixed medullaryfollicular and medullary-papillary carcinoma of the thyroid: one or two entities? Zentralbl Pathol 139: 333-335, 1993.

4) Parker LN, Kollin J, Wu SY, Rypins EB, Juler GL. Carcinoma of the thyroid with a mixed medullary, papillary, follicular, and undifferentiated pattern. Arch Intern Med 145: 1507-1509, 1985.

5) Pastolero GC, Coire CI, Asa SL. Concurrent medullary and papillary carcinomas of thyroid with lymph node metastases. A collision phenomenon. Am J Surg Pathol 20: 245-250, 1996. 